\title{
Voir, savoir et devenir médecin
}

Devenir soignant ou médecin relève d'apprentissages théoriques et pratiques. Voir et savoir. Depuis deux cents ans, la médecine moderne s'est construite sur des preuves visibles, objectives et reproductibles. À l'origine de cette médecine factuelle se trouve la pratique systématique de l'autopsie.

L'autopsie, plus que tout autre acte médical, peut être considérée comme l'incarnation même de la constitution d'un savoir objectivé sur le corps humain. Pour celui ou celle qui est en passe de devenir un professionnel de soins, l'autopsie et la dissection sont aussi une initiation au monde médical des souffrances et un point de cristallisation des rapports complexes et parfois ambigus entre les médecins, les patients et le public en général. Dès leur apparition au XIX ${ }^{\mathrm{e}}$ siècle, les codes de bonne conduite médicale ne s'y trompent pas et ce qui devient un droit appelle aussitôt des devoirs ${ }^{[1]}$. L'acte de la dissection est encadré par la nécessité absolue de règles précises et d'une démarche systématique. Plus rares sont les réflexions et les analyses qui s'interrogent sur le vécu de ceux et de celles qui traversent ces apprentissages-initiations. Aussi, la contribution d'Angélique Bonnaud-Antignac et de ses collaborateurs, qui paraît dans ce numéro, doitelle être examinée avec un intérêt particulier ${ }^{[2]}$.

L'autopsie constitue souvent la première rencontre des étudiant(e)s en médecine avec la maladie et la mort. L'autopsie, et plus généralement la pratique de la médecine, n'est pas seulement l'établissement d'une autorité professionnelle sur la vie. Souffrance et soins, connaissance et pouvoir médical se conçoivent et se construisent aussi à l'échelle d'un individu qui cherche à établir un rapport juste avec la souffrance et la mort d'autrui. Le passage dans une salle de dissection est à cet égard une expérience existentielle clé dans la construction d'une identité professionnelle personnelle.

«Ouvrez quelques cadavres, vous verrez aussitôt disparaître l'obscurité que jamais la seule observation clinique n'aurait pu dissiper », affirme Xavier Bichat (1771-1802) en 1801. La pratique de l'autopsie s'impose définitivement en Occident avec la première révolution de la pensée biologique au moment de la Renaissance, considérant qu'une pensée médicale rationnelle devrait s'orienter vers ce qui est reproductible, selon une régularité qui justifiera l'extension de la notion de loi à la nature. La dissection, d'abord purement anatomique - pour connaître les structures du corps humain - deviendra progressivement, aux $\mathrm{XVII}^{\mathrm{e}}$ et $\mathrm{XVIII}{ }^{\mathrm{e}}$ siècles, dissection anatomo-pathologique. Méthodique et systématique, elle dresse pendant deux cents ans l'inventaire complet et précis des lésions du corps d'un défunt.

Il s'agit de voir. De voir en détail, minutieusement et méthodiquement. Le regard médical scrute les structures d'un corps humain. Il mesure, pèse, toise et note, d'abord sur un tableau mural et ensuite dans un rapport. Il s'agit d'une manière de regarder et de voir le corps. Mais voir après, après la mort. L'exercice peut énoncer, mettre un terme définitif sur une disparition et il peut servir pour d'autres à l'avenir.

La véritable valeur scientifique de ce « voiraprès » réside dans sa confrontation avec un autre regard, le regard clinique. L'invention de la confrontation des deux regards fonde véritablement l'acquis de la médecine dite «rationnelle» des $\mathrm{XIX}^{\mathrm{e}}$ et $\mathrm{XX}^{\mathrm{e}}$ siècles. Elle télescope le temps d'avant et d'après, mais avec l'exigence que la connaissance acquise servira uniquement dans le futur et pour la 
formation des soignants et des médecins. Le regard clinique cherche dans la multiplicité des plaintes des malades et des impuissances des corps des traces ou des signes qui témoignent de la localisation et de la nature des lésions. Du choc des données de l'autopsie et de la clinique naît la compréhension de ce qu'on définira comme maladie.

$\mathrm{Au} \mathrm{XIX}^{\mathrm{e}}$ et $\mathrm{XX}^{\mathrm{e}}$ siècles, le recours de plus en plus aisé et systématique à l'autopsie permet de comparer les lésions de malades décédés des suites de signes cliniques similaires et de corréler signes et lésions organiques d'une même personne avant et après sa mort. De la capacité à voir et voir beaucoup, dépend la valeur d'un médecin expérimenté. En effet, Claude Bernard note déjà au milieu du $\mathrm{XIX}^{\mathrm{e}}$ siècle «qu'on n'arrivera jamais à des généralisations fécondes et lumineuses sur les phénomènes vitaux qu'autant qu'on n'aura expérimenté soi-même et remué dans l'hôpital, l'amphithéâtre ou le laboratoire, le terrain fétide et palpitant de la vie ${ }^{[2]}$.

Ce qui est une exhortation à la connaissance pour les uns, peut être, pour l'apprenti soignant et médecin, un face-à-face initial avec la mort. Ce que chaque dissection rappelle à l'enseignant et à l'enseigné, c'est que même au bout d'une quête scientifique et objective, le sujet et le ressenti ne disparaissent jamais totalement. Tout comme les étudiants interrogés par Angélique Bonnaud-Antignac, le professeur d'anatomie ou d'anatomie pathologique et son assistant se protègent par une distance affective établie dans le cadre de leur profession.

En septembre 1994, dans un article du journal Le Monde, Jean-Yves Nau, après avoir suivi des étudiants en médecine dans leur formation, constate : « Secret des corps, avant toute chose. Ces corps qui se dénudent quand l'ordonnent les blouses blanches, ces corps perclus de souffrance qui glissent vers leur fin. Certaines images ne me quitteront plus, écrit celui qui n'a pas subi les brutalités du bizutage carabin dont l'archaïsme a, entre autres fonctions, celle d'aider à oublier ces mêmes images. Le souvenir de certaines peaux, celui d'insupportables odeurs suivra aussi longtemps l'auteur. Secret des corps fermés, mystère des organismes ouverts. » Mais que savons nous du vécu des étudiants en train de devenir soignants ou médecins?

Lorsqu'en première année d'études médicales on pousse pour la première fois la lourde porte d'un institut d'anatomie et d'anatomie-pathologique, il y a comme un pressentiment. Ici s'arrête l'atmosphère calfeutrée des cours théoriques d'un amphithéâtre. Ici commence la vie médicale. Il s'agit d'une initiation et non plus d'une formation. Ici on devient apprenti médecin ou soignant. Le choc est rude, les odeurs sont âpres, les images poursuivent l'impétrant attiré par les secrets de la vie et du corps humain et le lieu exerce sur lui une indéniable fascination. En même temps, il crée un malaise et repousse. Attirance et répulsion. Attente. Savoir. Voir.

L'historien de la médecine américain John Harley Warner mène depuis des années une enquête sur le passé et le présent de la construction de l'identité professionnelle des soignants et des médecins. Des images parlent de manière éloquente des difficultés des étudiants de venir à terme avec l'expérience de la dissection depuis au moins un siècle.

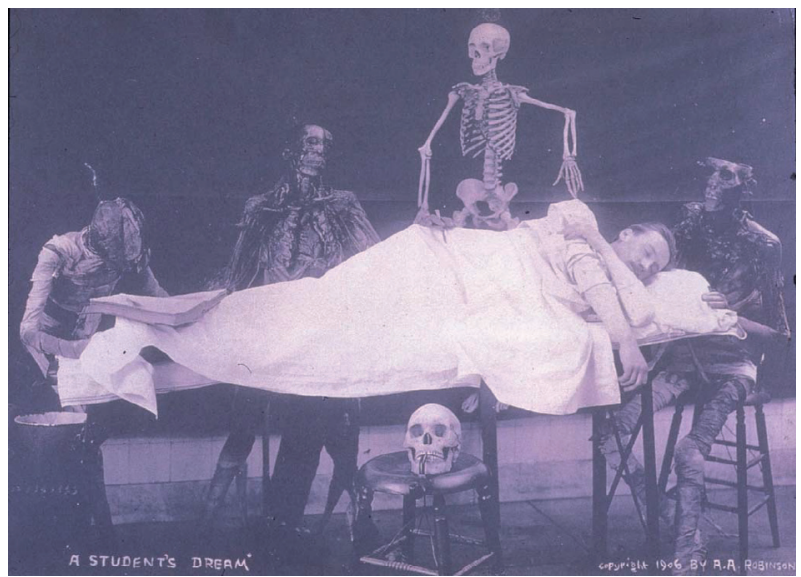

Autopsie. Voir par soi-même. Voir la facture et la constitution réelle d'un corps humain. Voir pour apprendre. Voir pour connaître. Voir pour devenir médecin. Voir à l'intérieur d'un corps humain. Le choc tient au fait qu'on se voit aussi soi-même. Ce déplacement tient bien de l'initiation. Déplacement vers un lieu où les corps s'ouvrent. L'initiation fait perdre l'innocence, nul n'entre ici qui ne 


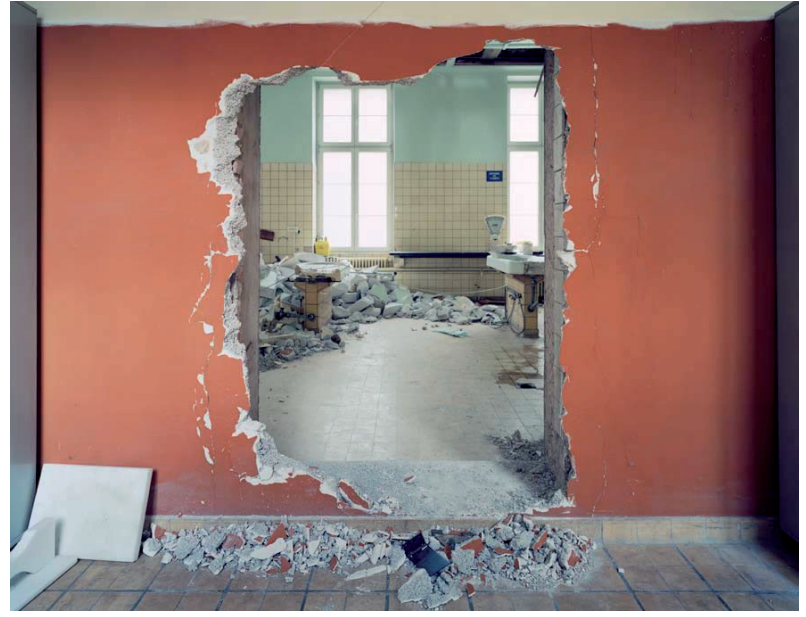

sera médecin ou soignant. Personne ne sort indemne de cette rencontre. Devant la charge émotionnelle du lieu, il paraît étonnant que la formation médicale n'y prête pas une attention particulière d'encadrement telle qu'elle s'esquisse dans les conclusions de la contribution d'Angélique Bonnaud-Antignac.

La salle de dissection est un lieu qui affirme avec force que le médecin passe son existence à regarder la souffrance et la mort en face. Un tel face-àface peut être atroce, au point qu'on ne le supporte pas sans défense, sans rites et sans un encadrement strict. Dans l'aboutissement d'une tradition philosophique dominée par le cartésianisme, le corps est devenu un objet de recherches scientifiques. Michel Foucault affirme qu'il a fallu passer par le moment de la mort pour constituer en objet le support matériel de notre existence. Le corps devient ainsi matière à investiguer.

Nous sommes aujourd'hui au début du $\mathrm{XXI}^{\mathrm{e}}$ siècle au seuil d'une métamorphose de cette tradition. Des instituts d'anatomie pathologique, comme le plus ancien des deux services d'anatomiepathologique de la faculté de médecine de Strasbourg, ferment définitivement leurs portes. Les autopsies deviennent de plus en plus rares en pathologie et les analyses histologiques se sont banalisées, au point de ne plus requérir systématiquement un laboratoire universitaire. Un travail récent du photographe Pierre Filliquet en collaboration avec l'auteur de cet éditorial témoigne

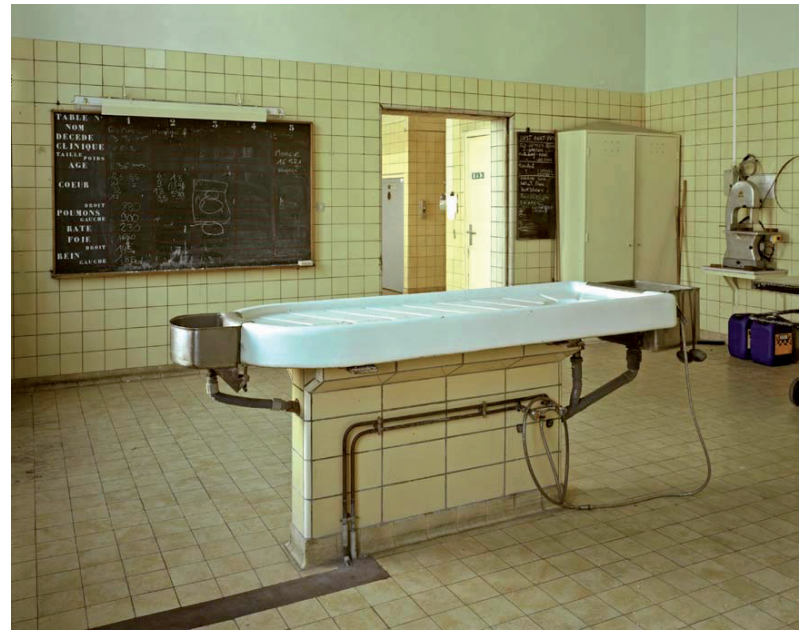

de la disparition, après presque 130 ans d'activité ininterrompue au «service de la science médicale », de salles d'autopsie désaffectées de la faculté de médecine de Strasbourg ${ }^{[4]}$. Des salles qui témoignent silencieusement de la routine pratique, professionnelle et perspicace d'arracher aux corps leurs derniers secrets, les causes du décès.

La médecine moderne se déconstruit au fur et à mesure que la médecine contemporaine se recompose. Le modèle séculaire de la méthode anatomoclinique est dépassé par les moyens contemporains d'imagerie médicale qui permettent désormais de scruter le corps du temps de son vivant. Plus besoin d'attendre la mort pour voir. Plus besoin d'ouvrir les corps pour voir. Dans certaines circonstances «l'usager de soins » peut même voir l'intérieur de son propre corps du temps de son vivant, sur des écrans endoscopiques et opératoires. Les approches de la biologie subcellulaire et moléculaire permettent, de plus en plus tôt, d'identifier des modifications génétiques qui prédisposent à l'apparition d'une maladie donnée. La médecine est passée d'un raisonnement où «on sait après ce qui était là avant » à celui où « on sait avant ce qui sera là après ». L'autopsie comme pratique constitutive d'une construction de savoirs médicaux est reléguée au statut d'un élément d'histoire médicale. La formation médicale, peut-être à tort, considère que sa valeur pédagogique ne justifie plus son maintien à échelle systématique. 
Le cadre de référence du regard médical change. Ce que nous perdons au seuil du $\mathrm{XXI}^{\mathrm{e}}$ siècle c'est le contact physique avec le corps. Nous perdons aussi un face-à- face avec la mort, difficile mais constitutif de l'identité du médecin. Et nous perdons la structuration du temps de la maladie par un avant et un après. Désormais le temps médical peut se superposer et s'inverser.

Il peut paraître incongru, au moment où les autopsies et la dissection sont en forte diminution dans la pratique médicale et dans la formation des soignants, de s'interroger sur leur retentissement psychologique sur ceux et celles qui y participent. Mais, à y regarder de plus près, peut-être en va-t-il de la dissection comme il en va de la santé en général : nous commençons seulement à y prêter attention lorsque nous sommes en passe de les perdre. Il paraît plus que légitime et souhaitable de s'interroger sur le vécu des étudiants à l'égard de leur formation et d'intégrer ces réflexions dans la conception de la formation elle-même. Mais il serait temps également d'interroger plus en détail la frontière entre le réel et le virtuel dans la formation médicale, à l'aube d'un siècle qui voit s'affirmer le second aux dépens du premier. Rappeler dans ce contexte que l'autopsie et la dissection ne sont pas seulement un apprentissage pratique mais aussi, depuis 200 ans, un moment de la construction d'une identité professionnelle revient aussi à constater que les sciences humaines et sociales peuvent être, dans la formation des futurs professionnels de santé, une démarche qui va bien au-delà de la simple transmission d'une culture générale, quelle que soit la valeur qu'on accorde par ailleurs à celle-ci.

\section{Christian BONAH}

Professeur d'Histoire des sciences de la vie et de la santé

Faculté de médecine de Strasbourg Membre junior de l'institut universitaire de France. Président du Collège des enseignants de sciences humaines et sociales en faculté de médecine. Mailto : christian.bonah@medecine.u-strasbg.fr

\section{Références}

1. Bonah C. L'expérimentation humaine. Discours et pratiques en France, 1900-1940. Paris : Les Belles Lettres, 2007:92-112.

2. Bonnaud-Antignac A, Armstrong O, Hamel A, Rogez J-M, Guilloton D, Maugars Y et al. Le vécu des dissections au laboratoire d'anatomie. Conséquences psychologiques et actions pédagogiques. Pédagogie Médicale 2008;9:201208.

3. Bernard $\mathrm{C}$. Introduction à la médecine expérimentale. Paris : Baillière, 1865:28.

4. Filliquet P, Bonah C. Autopsies. Paris : Micrografikeditions, 2008 (à paraître). 